\title{
The success rate of posterior tibial nerve block in the ankle with and without ultrasound guidance: $A$ clinical trial study for pain management in emergency departments
}

\section{Behrang Rezvani Kakhki ${ }^{1}$, Mohsen Ebrahimi ${ }^{1}$, Mahdi Foroughian $^{1}$, Samaneh Khajeh Nasiri $^{2}$, Vahid Eslami $^{3}$, Saeideh Anavri Ardakani ${ }^{4}$, Sayyed Reza Ahmadi ${ }^{*}$}

'Department of Emergency Medicine, Faculty of Medicine, Mashhad University of Medical Sciences, Mashhad, Iran 2Department of Radiology, Faculty of Medicine, Mashhad University of Medical Sciences, Mashhad, Iran

${ }^{3}$ University of Texas Health Science Center at San Antonio, Texas, USA

${ }^{4}$ Department of Neurology, Mashhad University of Medical Sciences, Mashhad, Iran

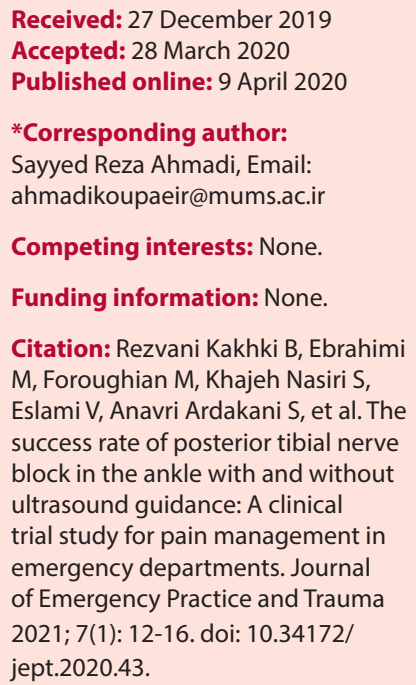

\begin{abstract}
Objective: The purpose of the current study was to assess the success rate of posterior tibial nerve block in the ankle with and without ultrasound guidance for pain management in emergency departments.

Methods: This clinical trial was conducted on 80 individuals who needed posterior tibial nerve block in the ankle at the emergency department of Hashemi Nejad hospital and Edalatian emergency center in Mashhad, Iran. The eligible individuals were randomly assigned to one of two groups, designated the control (landmark-based nerve block) and the case group (ultrasound-guided nerve block). The two groups were compared in terms of the main measurable outcomes. The data were analyzed using SPSS software (version 20) by nonparametric tests.

Results: According to the findings, the mean and median of nerve block success in the landmark-based and ultrasound-guided methods were significantly different between the two groups, both $15(P=0.02)$ and $30(P=0.001)$ min post-intervention. In this regard, nerve block with ultrasound guidance had a higher success rate compared to the landmark method. However, no significant difference between the two interventions was found in terms of the mean and median of the procedure duration $(P=0.8)$ and injection frequency $(P=0.4)$. On the other hand, the two groups were significantly different regarding the median and mean of patient satisfaction $(P=0.00)$, duration of analgesia $(P=0.004)$, and nerve block-related complications $(P=0.03)$.

Conclusion: The findings revealed that the relatively new technique of nerve block by ultrasound-guide resulted in better outcomes than the landmark-based method. Consequently, this method could be adopted to control acute pain in the emergency departments and improve patient care.

Keywords: Nerve block, Tibial nerve, Ultrasound, Ankle
\end{abstract}

\section{Introduction}

Pain is an unpleasant experience that occurs due to potential tissue damage. Since the goal of medical science is to maintain and improve health and reduce suffering, effective pain relief is a matter of fundamental importance (1). A nerve block refers to the induction of any deliberate interruption in the traveling of the signal throughout the nerve, usually performed with the aim of reducing pain. Nerve block is a short-term process that usually lasts from several hours to days, during which local anesthetic, corticosteroid, and other substances are injected into or near the nerve (2). This medical intervention has many applications in the emergency department and outpatient procedures, such as temporary pain relief (3). According to the literature, the landmark-based nerve block not only has a high probability of block failure, but also is associated with more complications (4). The peripheral nerve localization methods (e.g., nerve locator and nerve stimulator) and injection techniques are very helpful in the localization of the nerve. However, they do not display 
the needle path upon entering the skin until reaching the nerve and result in repeated attempts to find the nerve. This not only leads to a considerable loss of time, but also causes pain and possible complications in patients (5) and cannot reliably prevent intraneural injection (6). Moreover, blocking through these methods will be much more difficult in cases with disturbed local anatomy (e.g., history of surgery, radiotherapy, mass, obesity, and muscle hypertrophy) (7). When using these methods, it is not reliable to depend on clinical signs, such as severe pain upon local anesthetic injection. Accordingly, there are several pieces of evidence reporting a lack of pain during injection, followed by nerve injury events (8-11). Although the use of injection pressure monitoring is very useful, it cannot preclude the occurrence of intraneural injection (12). Ultrasound-guided nerve block has been commonly employed worldwide for several years. Several articles have been published in this regard in anesthetic journals (4,13-15). This method has been reported to have higher effectiveness than the previous landmark-based blocking methods (16-17). Currently, ultrasonography is available in the majority of hospitals; therefore, it can be commonly used for nerve blocking. However, little is known about the superiority of this method over the previous traditional techniques. In a study conducted by Perlas et al, the use of ultrasound-guided sciatic nerve block was demonstrated to result in greater success, faster onset of the effect, and better enhancement of sensory motor block without prolonging the implementation time or any complications (18). In another study carried out by Kapral et al, arm nerve block with ultrasound guidance led to a greater degree of block success, compared to the nerve stimulation method (19). However, in a systematic review performed by Liu et al, although the use of ultrasound reduced the duration and frequency of analgesia, it showed no significant difference with the traditional methods (20). Given the fact that all investigations reviewed in the mentioned study had a small sample size and were in the form of case reports, the authors recommended to perform further research, especially randomized controlled trials, to obtain more accurate findings. Bearing this in mind, the present study was conducted to compare the success rate of posterior tibial block in the ankle with and without ultrasound guidance for pain management in the emergency departments.

\section{Methods}

This clinical trial was conducted on 80 patients referring to the emergency department of Hashemi Nejad hospital and Edalatian Emergency Center in Mashhad, Iran from 2014 to 2015.

The inclusion criteria were: 1) isolated trauma in the posterior tibial nerve in the foot and toes, 2) age over 18 years and 3) no previous deformities in the ankle. On the other hand, the patients with multiple traumas and injury to the ankle and those receiving analgesics for any cause in advance were excluded from the research.

After obtaining informed consent from the eligible individuals, they were divided into two groups of control (i.e., landmark-based nerve block) and intervention (i.e., ultrasound-guided nerve block) using closed envelopes, randomly. Then, the two groups were compared in terms of the main measurable outcomes. Given that the patients were in a prone position, they were not aware of the type of intervention. All patients were subjected to ultrasound using the Honda 2000 ultrasound device with a 7.5 MHZ linear probe.

The time required to perform nerve blocking was considered from the onset of finding the correct point for the needle insertion until the needle removal after ensuring the nerve block. The rate of blocking success was estimated 15 and 30 minutes after the onset of the procedure. This rate was determined based on the number of changes in the needle position for blocking, quality of the block (based on patient rating), duration of postblocking analgesia (using visual analogue scale [VAS] as having a score of $<3$ showing the lowest analgesia), patient satisfaction (using VAS with one and 10 showing the lowest and highest amount of satisfaction, respectively), unwanted complications (e.g., hematoma, injection site pain, paresthesia, and dystocia), and frequency of attempts to create local anesthesia.

To examine the sensory block, after the end of the nerve block, the patients were positioned in supine and monitored for 60 minutes by an emergency medicine resident blinded to the study. The resident checked the loss of sensation every 5 minutes upon the injection. In this regard, they examined five specified areas using pinprick and cold test. Lack of stimulation was considered as a complete sensory block. To examine the motor block, after the end of the nerve block, the patient was placed in a supine position and monitored every 5 minutes upon injection for 60 minutes in terms of motor block by an emergency medicine resident blinded to the study. After the completion of the motor and sensory block, the following two questions were asked from each patient: 1) raise the foot in which you feel more numbness and 2) express your satisfaction with this procedure on a range of 1-10 based on VAS.

The obtained information was recorded in a form by a resident blinded to the study using codes, and then delivered to a statistical specialist for analysis. All nerve blocks were performed by a radiologist. The patients received no intravenous or oral sedation during the interventions. They were asked about the emergence of complications within $48 \mathrm{~h}$ post-intervention.

The data were entered in SPSS software (version 20) and analyzed. Descriptive statistics (e.g., frequency) were used to show the results. Nonparametric tests like MannWhitney U, and Kruskal-Wallis were used to compare continuous variables between groups; considering $P$ value less than 0.05 , as statistically significant. 


\section{Results}

Out of the 80 patients, $59(73.7 \%)$ cases were males. The mean age of the patients was $34.48 \pm 10.51$ years (Age range: above 18 years). Most of the patients were within the age range of $26-34(32.5 \%)$, and the lowest age group was over 50 years $(7.5 \%)$. The control group subjected to landmark-based nerve block consisted of 30 males and 40 females. The results of the statistical test demonstrated no significant difference between the males and females in the control group in terms of the mean of blocking success $(P=0.2)$. The case group undergoing nerve block through ultrasound guidance included 29 males and 11 females. Similarly, blocking success showed no significant difference between the males and females in this group $(P=0.1)$.

As shown in Table 1, comparison of blocking success between the patients subjected to landmark-based and ultrasound-guided nerve block methods 15 minutes postintervention showed a significant difference between the two groups in this regard $(P=0.02)$. Accordingly, the case group had a lower mean value of an unsuccessful nerve block, compared to the control group.

As shown in Table 2, the results also revealed that in the case of the nerve blocking success 30 min post-intervention, the mean of unsuccessful nerve block occurrence was significantly lower in the ultrasound-guided group in comparison to the landmark group $(P=0.001)$.

Comparison of the procedure duration between the landmark-based and ultrasound-guided nerve block methods indicated no significant difference $(P=0.8)$. Likewise, no statistically significant difference was observed between the two groups in terms of injection frequency $(P=0.4)$. Additionally, we observed a substantial difference in average patient satisfaction between the control and the case group $(3.67 \pm 0.87$ vs. $3.54 \pm 0.98$; $P=0.02)$. The ultrasound-guided nerve block resulted in a higher level of patient satisfaction compared to the landmark-based method.

Based on the results, the length of the analgesia was significantly different with patients undergoing landmark- based technique $(2.00 \pm 0.00)$ and those undergoing the ultrasound-guided method $(2.66 \pm 1.15) \quad(P=0.004)$. In this respect, in the case group the mean length of the analgesia was considerably higher than the control group $(P=0.004)$. As depicted in Table 3 , there was a significant difference between the two groups in terms of complications. In this regard, the patients in the case group had a lower complication rate as compared to the control group $(P<0.05)$.

\section{Discussion}

A successful nerve block requires the exact localization of the nerve, proper needle guidance, and suitable amount of local anesthetic application. The conventional methods (e.g., landmark-based technique) are performed blindly and therefore cause discomfort and complications. The use of ultrasound guidance in nerve blocking can increase the efficiency of this intervention and reduce the complications (21). A successful and safe implementation of the peripheral nerve block under ultrasound guidance requires the adoption of an appropriate imaging technique for the detection of the desired nerve, skillful guidance of the needle in a coordinated manner, and evaluation of the drug diffusion in the appropriate site. Our results revealed a significant difference between the patients undergoing ultrasound-guided never block and those subjected to the landmark-based method in terms of blocking success both 15 and 30 minutes post-intervention. The rate of the unsuccessful nerve block was lower in the ultrasoundguided group compared to the landmark groups. One of the benefits of ultrasound is the reduction of the chance of failure and minimal effective dose (22-24). The drug diffusion site in an intratissue injection is well visualized in a hypo echoic image. This facilitates the accurate injection of the drug in the desired location. Moreover, this technique prevents intravascular injections, thereby reducing the likelihood of systemic toxicity with anesthetic drugs. Since the introduction of ultrasound as a technology for local anesthesia, the use of this method has gained great popularity due to its simplicity and

Table 1. Comparison of nerve blocking success between the patients undergoing landmark-based and ultrasound-guided methods 15 minutes post-intervention

\begin{tabular}{llll}
\hline & Landmark (Control) & Ultrasound (Case) & P value \\
\hline Successful sensory block & $1.81 \pm 0.66$ & $2.00 \pm 0.85$ & 0.02 \\
Successful sensory and motor block & $1.25 \pm 0.46$ & $1.47 \pm 0.79$ & 0.001 \\
\hline Unsuccessful block & $1.30 \pm 0.48$ & $1.00 \pm 00$ & 0.01 \\
\hline
\end{tabular}

Table 2. Comparison of nerve blocking success between the patients undergoing landmark-based and ultrasound-guided methods 30 minutes post-intervention

\begin{tabular}{llll}
\hline & Landmark (Control) & Ultrasound (Case) & P value \\
\hline Successful sensory block & $2.05 \pm 0.40$ & $2.17 \pm 0.52$ & 0.01 \\
Successful sensory and motor block & $1.07 \pm 0.27$ & $1.42 \pm 0.81$ & 0.001 \\
Unsuccessful block & $1.50 \pm 0.53$ & $1.00 \pm 0.00$ & 0.001 \\
\hline
\end{tabular}


Table 3. Comparison of complication rates between landmark-based and ultrasound-guided nerve block methods

\begin{tabular}{lccc}
\hline & $\begin{array}{c}\text { Landmark } \\
\text { (Control) }\end{array}$ & $\begin{array}{c}\text { Ultrasound } \\
\text { (Case) }\end{array}$ & P value \\
\hline Hematoma & $4.00 \pm 0.00$ & $3.33 \pm 1.15$ & 0.001 \\
Paresthesia & $1.00 \pm 0.00$ & $4.00 \pm 0.00$ & 0.003 \\
Pain & $4.00 \pm 0.00$ & $2.00 \pm 0.00$ & \\
No complications & $3.67 \pm 0.87$ & $3.54 \pm 0.98$ & 0.02 \\
\hline
\end{tabular}

precision $(3,18,25)$. Ultrasonography is currently available in many hospitals; as a result, it can be commonly used for nerve blocking. Comparison of complications between the landmark-based and ultrasound-guided methods revealed a significant difference. In this regard, the mean of the non-complicated nerve block was lower in the ultrasound-guided group than in the landmark group $(P=0.02)$. One of the most important merits of ultrasound guidance is the reduction of block-related complications. There are various reports regarding these complications (26). For instance, Bigeleisen reported a nerve damage probability of $0.002 \%$ in patients subjected to ultrasoundguided block (27). Furthermore, Chan et al observed an increase in nerve diameter following intraneural injection. They reported that stopping injection in both of the investigated cases prevented the incidence of neurologic complications (13). Certain findings of this study revealed that the length of analgesia between the case and the control groups was significantly different. In this respect, the patients undergoing nerve block with ultrasound guidance had a longer duration of analgesia. In line with our findings, Hadzic et al concluded that ultrasound-guided nerve block triggered early analgesia and increased its duration. Despite the disparity between nerve blocks anatomy and physiology, they reported that ultrasound resulted in a $29 \%$ decrease in the block onset time and a $25 \%$ increase in the duration of analgesia (28). According to Marhofer and Chan, the nerve block under ultrasound guidance requires a lower anesthetic volume and provides sufficient safety for regional anesthesia, compared to nerve stimulation $(13,23)$. Moreover, Wegener et al reported that the localization of the nerve was $10 \%$ more accurate by ultrasound compared to a nerve stimulator (6). van Geffen et al also indicated that the patients receiving peripheral nerve block had lower resting and moving pain scores, compared to those undergoing peripheral nerve block (7). Mariano et al showed that the ultrasound-guided nerve block resulted in a higher rate of pain relief and lower vascular damage in comparison to nerve stimulation method (8). In line with these results, our findings indicated that nerve block with ultrasound guidance led to longer pain relief than the landmarked-based method. Finally, the comparison of patients' satisfaction with landmark-based and ultrasound-guided methods showed a significant difference between the two groups. In this regard, the patients undergoing nerve block with ultrasound guidance reported a higher level of satisfaction than those subjected to the landmark-based method $(P=0.000)$. In general, the benefits of ultrasound-guided peripheral nerve block method include better nerve localization that reduces the time to perform blocking, less local anesthetic volume, better visualization of anesthetic drug diffusion, lower risk of drug toxicity, faster block onset, more complete nerve block, more prolonged sensory and motor nerve block, fewer complications, and higher patient satisfaction (29).

\section{Limitations of the study}

Taking into account that all blocking procedures were performed by a radiology specialist, the skill level of the radiologist may have partly affected the results of the research. A further limitation of this research was the inability of some patients to comply which were omitted from our study.

\section{Conclusion}

As the results of this study have demonstrated, ultrasoundguided nerve block, as a relatively new method, improved the quality of nerve blocking. Consequently, this technique can be employed to control the acute pain in the emergency department and improve patient care.

\section{Authors' contributions}

All authors met the four criteria for authorship contribution based on recommendations of the International Committee of Medical Journal Editors. $\mathrm{BRK}, \mathrm{ME}, \mathrm{MF}$, and VE contributed to study design and protocol development, sampling and data collection. SKN, VE, SAA, and SRA contributed to sampling, data analysis and data collection. All authors contributed in manuscript writing and revisions.

\section{Ethical issues}

The protocol of the study was approved by ethics committee of Mashhad University of Medical Sciences and researchers adhered to confidentiality of the evaluated files (IR.MUMS.fm.REC.1395.234). Additionaly, the study was registred at the Iranian Registry of Clinical Trials website (identifier: IRCT2017041733477N1; https://www.irct.ir/ trial/25807).

\section{Acknowledgements}

The authors would like to extend their gratitude to the authorities of Hashemi Nejad Hospital and Edalatian Emergency Center, affiliated to the Research Deputy of Mashhad University of Medical Sciences, Mashhad, Iran, for their cooperation. We would like to thank the Clinical Research Development Unit of Peymanieh Educational and Research and Therapeutic Center of Jahrom University of Medical Sciences for editing this manuscript.

\section{References}

1. Benzon H, Raja SN, Fishman SE, Liu SS, Cohen SP. Essentials 
of Pain Medicine E-book. Elsevier Health Sciences; 2011.

2. Vrachatis AD, Alpert MA, Georgulas VP, Nikas DJ, Petropoulou EN, Lazaros GI, et al. Comparative efficacy of primary angioplasty with stent implantation and thrombolysis in restoring basal coronary artery flow in acute ST segment elevation myocardial infarction: quantitative assessment using the corrected TIMI frame count. Angiology 2001; 52(3): 161-6. doi: $10.1177 / 000331970105200301$.

3. Gray AT. Ultrasound-guided regional anesthesia: current state of the art. Anesthesiology 2006; 104(2): 368-73. doi: 10.1097/00000542-200602000-00024.

4. Blaivas M, Lyon M. Ultrasound-guided interscalene block for shoulder dislocation reduction in the ED. Am J Emerg Med 2006; 24(3): 293-6. doi: 10.1016/j.ajem.2005.10.004.

5. Chan VW. Applying ultrasound imaging to interscalene brachial plexus block. Reg Anesth Pain Med 2003; 28(4): 340-3. doi: 10.1016/s1098-7339(03)00189-5.

6. Wegener JT, Boender ZJ, Preckel B, Hollmann MW, Stevens MF. Comparison of percutaneous electrical nerve stimulation and ultrasound imaging for nerve localization. Br J Anaesth 2011; 106(1): 119-23.

7. van Geffen GJ, van den Broek E, Braak GJ, Giele JL, Gielen MJ, Scheffer GJ. A prospective randomised controlled trial of ultrasound guided versus nerve stimulation guided distal sciaticnerve block at the popliteal fossa. Anaesth Intensive Care 2009; 37: 32-7.

8. Mariano ER, Loland VJ, Sandhu NS, Bishop ML, Lee DK, Schwartz AK, et al. Comparative efficacy of ultrasoundguided and stimulating popliteal-sciatic perineural catheters for postoperative analgesia. Can J Anaesth 2010; 57: 919-26.

9. Avellanet M, Sala-Blanch X, Rodrigo L, Gonzalez-Viejo MA. Permanent upper trunk plexopathy after interscalene brachial plexus block. J Clin Monit Comput 2016; 30(1): 51-4. doi: 10.1007/s10877-015-9681-z.

10. Shinn HK, Kim BG, Jung JK, Kwon HU, Yang C, Won J. Prolonged hemidiaphragmatic paresis following continuous interscalene brachial plexus block: a case report. Medicine (Baltimore) 2016; 95(24): e3891. doi: 10.1097/ md.0000000000003891.

11. Kahn RL, Ellis SJ, Cheng J, Curren J, Fields KG, Roberts $\mathrm{MM}$, et al. The incidence of complications is low following foot and ankle surgery for which peripheral nerve blocks are used for postoperative pain management. HSS J 2018; 14(2): 134-42. doi: 10.1007/s11420-017-9588-y.

12. Claudio R, Hadzic A, Shih H, Vloka JD, Castro J, KoscielniakNielsen Z, et al. Injection pressures by anesthesiologists during simulated peripheral nerve block. Reg Anesth Pain Med 2004; 29(3): 201-5. doi: 10.1016/j.rapm.2003.12.013.

13. Chan VW, Perlas A, Rawson R, Odukoya O. Ultrasoundguided supraclavicular brachial plexus block. Anesth Analg 2003; 97(5): 1514-7. doi: 10.1213/01. ane.0000062519.61520.14.

14. De Andrés J, Sala-Blanch X. Ultrasound in the practice of brachial plexus anesthesia. Reg Anesth Pain Med 2002; 27(1): 77-89.

15. Hua D, Wang Y, Ji S, Zha J, Xia Y. Ultrasound-guided brachial plexus block: comparison between inter scalene and supraclavicular approach. J Med Imaging Health Inform 2019; 9(5): 951-5. doi: 10.1166/jmihi.2019.2658.
16. Williams SR, Chouinard P, Arcand G, Harris P, Ruel $\mathrm{M}$, Boudreault $\mathrm{D}$, et al. Ultrasound guidance speeds execution and improves the quality of supraclavicular block. Anesth Analg 2003; 97(5): 1518-23. doi: 10.1213/01. ane.0000086730.09173.ca.

17. Chan VW, Perlas A, McCartney CJ, Brull R, Xu D, Abbas S. Ultrasound guidance improves success rate of axillary brachial plexus block. Can J Anaesth 2007; 54(3): 176-82. doi: 10.1007/bf03022637.

18. Perlas A, Brull R, Chan VW, McCartney CJ, Nuica A, Abbas $S$. Ultrasound guidance improves the success of sciatic nerve block at the popliteal fossa. Reg Anesth Pain Med 2008; 33(3): 259-65. doi: 10.1016/j.rapm.2007.10.010.

19. Kapral S, Greher M, Huber G, Willschke H, Kettner S, Kdolsky R, et al. Ultrasonographic guidance improves the success rate of interscalene brachial plexus blockade. Reg Anesth Pain Med 2008; 33(3): 253-8. doi: 10.1016/j. rapm.2007.10.011.

20. Liu SS, Ngeow JE, Yadeau JT. Ultrasound-guided regional anesthesia and analgesia: a qualitative systematic review. Reg Anesth Pain Med 2009; 34(1): 47-59. doi: 10.1097/ AAP.0b013e3181933ec3.

21. Grau T, Fatehi S, Motsch J, Bartusseck E. [Survey on current practice of regional anaesthesia in Germany, Austria, and Switzerland. Part 2: use, success rates and techniques]. Anaesthesist 2004; 53(9): 847-55. doi: 10.1007/s00101-0040720-2.

22. Willschke H, Marhofer P, Bösenberg A, Johnston S, Wanzel O, Cox SG, et al. Ultrasonography for ilioinguinal/ iliohypogastric nerve blocks in children. Br J Anaesth 2005; 95(2): 226-30. doi: 10.1093/bja/aei157.

23. Mohamed KH, Abdelrahman KA, Elameer AN, Ali IH. Morphine as an adjuvant to local anesthetics in axillary brachial plexus block in forearm and hand surgery. J Curr Med Res Pract 2019; 4(2): 131-6. doi: 10.4103/jcmrp. jcmrp_35_19.

24. Sandhu NS, Maharlouei B, Patel B, Erkulwater E, Medabalmi P. Simultaneous bilateral infraclavicular brachial plexus blocks with low-dose lidocaine using ultrasound guidance. Anesthesiology 2006; 104(1): 199201. doi: 10.1097/00000542-200601000-00028.

25. Redborg KE, Antonakakis JG, Beach ML, Chinn CD, Sites $\mathrm{BD}$. Ultrasound improves the success rate of a tibial nerve block at the ankle. Reg Anesth Pain Med 2009; 34(3): 25660. doi: 10.1097/AAP.0b013e3181a343a2.

26. Andrew T. Ultrasound Guidance for Regional Anesthesia. In: Miller R.D. Miller's Anesthesia. 7th ed. California: Elsevier; 2010. p. 1675.

27. Bigeleisen PE. Nerve puncture and apparent intraneural injection during ultrasound-guided axillary block does not invariably result in neurologic injury. Anesthesiology 2006; 105(4): 779-83. doi: 10.1097/00000542-200610000-00024.

28. Hadzic A, Dilberovic F, Shah S, Kulenovic A, Kapur E, Zaciragic A, et al. Combination of intraneural injection and high injection pressure leads to fascicular injury and neurologic deficits in dogs. Reg Anesth Pain Med 2004; 29(5): 417-23. doi: 10.1016/j.rapm.2004.06.002.

29. Marhofer P, Chan VW. Ultrasound-guided regional anesthesia: current concepts and future trends. Anesth Analg 2007; 104(5): 1265-9, tables of contents. doi: 10.1213/01.ane.0000260614.32794.7b. 